\title{
PARA UMA RECONSTRUÇÃO ATRAVÉS DAS TIC, DESDE UMA PERSPECTIVA DIDÁTICA caso de estudo do SUAyED UNAM
}

Adriana Leticia Díaz Manrique

Resumo

O Sistema Universidad Abierta y Educación a Distancia (SUAyED) da Universidad Nacional Autónoma de México é uma modalidade de estudo na qual o uso das TIC faz parte do que Cobo (2011) chama de "aprendizagem invisível". Neste trabalho foram identificadas as dificuldades com que se deparam tanto os alunos quanto os professores do SUAyED, bem como as estratégias didáticas utilizadas para propiciar situações de aprendizagem por meio de uma plataforma. Para isso, foi realizado um questionário online com 521 alunos e a 79 professores. Mediante as técnicas de conveniência e de grupos extremos, foram entrevistados sete professores que, com seus depoimentos, permitiram identificar as estratégias didáticas utilizadas para fazer com que os alunos se apropriem da aprendizagem. É evidente também que a ausência de um Programa de Estudos específico para essa modalidade gera dificuldades no sistema não presencial.

Palavras-chave: TIC; estratégias didáticas; situações de aprendizagem.

\section{TOWARDS A RECONSTRUCTION OF ICT-MEDIATED EDUCATION FROM A DIDACTIC PERSPECTIVE SUAYED UNAM case study}

\begin{abstract}
The Open University and Distance Education System (SUAyED) of the National Autonomous University of Mexico is an educational modality where the use of ICT, according with Cobo (2011), is called "invisible learning". This research identified: the difficulties faced by teachers and students of SUAyED, as well as the didactic strategies used to promote learning situations through a virtual platform. To do this, an online questionnaire was applied to 521 students and 79 teachers. Using the techniques of convenience and extreme groups, seven teachers were interviewed who allowed identifying through their testimonies, the didactic strategies used to ensure that students take ownership of the learning. It is realized that the absence of a specific Curriculum for this modality causes difficulties in this non-face-to-face system.
\end{abstract}

Keywords: ICT; didactic strategies; learning situations.

\section{HACIA UNA RECONSTRUCCIÓN DE LA EDUCACIÓN MEDIADA POR TIC DESDE UNA PERSPECTIVA DIDÁCTICA caso de estudio SUAyED UNAM}

Resumen

El Sistema Universidad Abierta y Educación a Distancia (SUAyED) de la Universidad Nacional Autónoma de México, es una modalidad de estudio en la que el uso de las TIC forma parte de lo que Cobo (2011) llama, "un aprendizaje invisible". En este estudio se identificaron: las dificultades que enfrentan docentes y alumnos del SUAyED, así como las estrategias didácticas empleadas para propiciar situaciones de aprendizaje a través de una plataforma virtual. Para ello, se aplicó un cuestionario en línea a 521 alumnos y a 79 docentes. Mediante las técnicas de conveniencia y grupos extremos, se entrevistó a siete docentes que, 
a través de sus testimonios, permitieron identificar las estrategias didácticas empleadas para conseguir que los alumnos se apropien del aprendizaje. Se advierte que la ausencia de un plan de estudios exprofeso para esta modalidad propicia dificultades en este sistema educativo no presencial.

Palabras clave: TIC; estrategias didácticas; situaciones de aprendizaje.

\section{INTRODUCCIÓN}

La Pandemia del COVID-19 ha traído grandes aprendizajes para el mundo entero. Ha evidenciado la debilidad del ser humano, de su sistema de salud y de su sistema económico, y a ello se aúna la necesidad de reconstruir el sistema educativo. La enseñanza remota de emergencia mediada por tecnologías para la educación superior se colocó como la principal alternativa para salir avante en esta emergencia sanitaria.

En México, el sistema educativo no estaba preparado para transitar de la educación presencial a la educación mediada por tecnologías y no sólo por la falta de conectividad que padece este país; sino por la ausencia de una didáctica acorde al contexto de la educación superior. Tal es el caso del Sistema Universidad Abierta y Educación a Distancia (SUAyED) de la Facultad de Ciencias Políticas y Sociales (FCPyS) de la Universidad Nacional Autónoma de México (UNAM), donde se imparte educación en la modalidad a distancia, misma que García Aretio define como "[...] un sistema de enseñanza en el que el estudiante realiza su aprendizaje por medio de materiales didácticos previamente preparados, con un escaso contacto con los profesores" (GARCÍA, 1987) así, se desarrolla el conocimiento a través de las TIC, lo que Cobo (2011) llama, "un aprendizaje invisible", no obstante, la mayoría de las actividades que se realizan son reproducidas del ambiente educativo presencial.

Si bien no es el tema central de este artículo, es conveniente apuntar que durante la pandemia el trabajo mediante TIC, en el que se desarrolla el SUAyED desde hace más de tres lustros, ha permitido que el sistema presencial o escolarizado de la UNAM, adopte mecanismos de apoyo para transitar temporalmente durante esta crisis sanitaria, de aquí la relevancia de que las instituciones educativas reconozcan los cambios que demanda el trabajo didáctico, en la educación a distancia, potenciando el uso de las TIC.

En el SUAyED de la FCPyS se trabaja con plataformas virtuales y el plan de estudios de la licenciatura en Ciencias de la Comunicación comparte el modelo de asignaturas del sistema escolarizado, cada asignatura dispone de un sitio integrado en una plataforma web que presenta toda la información relacionada con el desarrollo del curso: identificación del docente, forma de trabajo, contenidos, actividades y criterios de evaluación.

No obstante, a decir de Sánchez (2019) se requiere "[...] atender la eficiencia terminal del SUAyED, ya que es muy baja, $[\ldots]$ además de que las licenciaturas de este sistema que se desarrolla en la modalidad a distancia han sido consideradas como de segunda, esto es, que tienen poca credibilidad y no se tiene información ni conocimiento sobre ellas".

Esta situación motiva el interés de identificar la problemática que registra la modalidad educativa a distancia de la UNAM, específicamente en la licenciatura en Ciencias de la Comunicación, que a la fecha cuenta con 15 años de operación. Para ello, se aplicó, en esta investigación un cuestionario en línea a 521 alumnos y a 79 docentes. Mediante las técnicas de conveniencia y grupos extremos, se entrevistó a siete docentes que, a través de sus testimonios, permitieron identificar las principales problemáticas a las que se han enfrentado alumnos y docentes que participan en esa modalidad, además, se identificaron las estrategias didácticas empleadas para conseguir que los alumnos se apropien del aprendizaje.

\section{EL USO DIDÁCTICO EN LAS TIC EN EL PROCESO EDUCATIVO}

Diversas voces de especialistas (PERRENOUD, 2004; GARCÍA, 2007, 2009; CABERO, 2014; ZAPATA, 2014) han manifestado que el uso de las TIC en la educación va más allá de ser una herramienta de apoyo, toda vez que puede potenciar el abordaje educativo. Este trabajo respalda la propuesta de Díaz 
Barriga $(2013,2017,2020)$ de emplear las TIC desde una perspectiva didáctica, toda vez que la investigación realizada ha detectado que no se ha trabajado lo suficiente sobre los cambios que demanda una planeación didáctica sobre qué significa transitar de una clase frontal a un modelo educativo de multimedios en la educación superior.

A partir del estado del arte que se llevó a cabo en esta investigación y que forma parte de un trabajo doctoral en educación, fue posible inferir que si bien el total de los autores consultados reconocen la necesidad de construir estrategias que permitan apuntar el estudio a través del uso de las tecnologías, hasta la realización de esta investigación no se registran estudios concretos que reporten resultados de estrategias didácticas aplicadas en la educación a distancia en programas educativos de nivel superior.

Para ello fue relevante identificar que, como lo dijera Freinet (1963), “[...] la situación de grupo escolar es trascendental para presentar propuestas acordes a cada situación didáctica". Estudiar a Freinet permitió ubicar una perspectiva didáctica, pero acorde a la modalidad educativa que se aborda, sin perder de vista las características de los estudiantes del siglo XXI y de los docentes de esta modalidad, considerando los avances tecnológicos, pero sobre todo, que el centro es el proceso educativo.

Para autores como Gerver, es necesario crear situaciones de aprendizaje reales para el alumno, a fin de que éste aprenda, considerando que el aprendizaje debe importar a quien aprende y para que importe debe estar dotado de relevancia y de contexto. De esta forma, "[...] el aprendizaje debe tener una naturaleza contextualizada que permita una conexión con la realidad, que le ayude a entender la vida fuera de la escuela" (GERVER, 2012), pero además de real, el aprendizaje debe ser relevante y para serlo debe conectar con algo que conoce o sabe el alumnado de su propia realidad (PRENSKY, 2011).

\section{METODOLÓGICA}

Esta investigación está centrada en la didáctica que emplean los docentes a distancia de la licenciatura de ciencias de la comunicación que forman parte del SUAyED de la FCPyS-UNAM, con el objetivo de analizar y documentar las prácticas docentes que se apoyan de las TIC. Aquí se analiza la didáctica que emplean docentes, empero, también se considera la opinión de los alumnos (ambos de la modalidad a distancia) por considerar que se trata de un binomio indisociable. En el SUAyED, los estudiantes se encuentran acompañados por un docente. “[...] Los docentes están capacitados para trabajar en la modalidad educativa a distancia; cuentan con una formación académica y experiencia en las asignaturas para conducir el aprendizaje conforme al plan de trabajo que han elaborado" (ANDRADE, 2014).

Se parte de las preguntas de investigación: ¿cuáles son los recursos didácticos que el docente del SUAyED emplea para propiciar situaciones de aprendizaje? y ¿qué dificultades han enfrentado para reconstruir una perspectiva didáctica acorde con un sistema de educación a distancia?

En el desarrollo de esta investigación, se emplea una metodología de corte cualitativo, que de acuerdo con Taylor y Bogdán (1984) “[...] sigue un diseño de investigación flexible, toda vez que en la metodología cualitativa el investigador ve el escenario y a las personas en una perspectiva holística y las personas, los escenarios o los grupos no son reducidos a variables, sino considerados como un todo".

\section{Procedimiento}

El desarrollo del estudio constó de dos fases: la primera de ellas consistió en la aplicación de dos cuestionarios diagnósticos dirigidos a docentes y alumnos. Consciente de que se trata de un estudio de corte cualitativo, se optó por el cuestionario para nutrir de información, considerando que se trata de la modalidad a distancia y se requería contactar primeramente a los sujetos de investigación e identificar las problemáticas que enfrenta el Sistema. Las respuestas que arrojaron los cuestionarios fueron empleadas para llevar a cabo la segunda fase, que consistió en elaborar un guión y aplicarlo en las entrevistas de manera directa a los docentes. 
Para la obtención de datos se emplearon las llamadas técnicas de conveniencia, pero aplicadas a la investigación cualitativa. Empleando la técnica de grupos extremos, que de acuerdo con Kleining (1994), "[...] se delimitan a submuestras donde estás difieren diametralmente una de otra". Para la construcción de los cuestionarios tanto para los alumnos como para los docentes, se requirió de un enfoque sistemático y organizado de las actividades a desarrollar (DOWNING, HALADYNA, 2006). El cuestionario integró 34 ítems para alumnos y 35 ítems para docentes. Los cuestionarios se encuentran disponibles en (DÍAZ, 2020).

\section{Aplicación}

Para validar los dos instrumentos de investigación se llevó a cabo una prueba piloto. Una vez realizada la aplicación del pilotaje y analizadas las preguntas y respuestas, se llevó a cabo la interpretación y mejoras de los instrumentos. Llevadas a cabo las modificaciones correspondientes al cuestionario y considerando que "[...] los estudios de corte cualitativo necesitan de una planeación minuciosa y cuidadosa antes de empezar con la recopilación de datos" (LANSKSHEAR Y KNOBEL, 2000) se elaboró un guión de preguntas que sirvió de base para el cuestionario que se aplicó a alumnos y docentes.

\section{FASE 1: CUESTIONARIOS ALUMNOS Y DOCENTES}

Los cuestionarios fueron aplicados a través de la herramienta de Google Forms@ y se registró una participación total de 521 alumnos y 79 docentes. Una vez compiladas las respuestas de los cuestionarios, se procedió a analizar la información almacenada en hojas de cálculo, siendo seleccionadas sólo aquellas respuestas que son útiles para esta investigación. Así, para responder a la metodología propuesta se consideraron las respuestas de la siguiente muestra:

Alumnos. Se eligió a los alumnos de tercer semestre debido a que tras haber cursado un año en el SUAyED cuentan con elementos que les permite opinar sobre el tema; asimismo, se determinó considerar a los alumnos del séptimo semestre por encontrarse a dos semestres de concluir su carrera profesional y contaban con mayores elementos para opinar sobre el desarrollo de sus estudios en esa modalidad. De esta forma se responde a la característica de los grupos extremos. Así, se consideraron las respuestas de un total de 165 alumnos de los cuales, 108 forman parte del tercer semestre y 57 del séptimo semestre.

Docentes. Se consideraron las respuestas de los 79 docentes participantes. Se trata de profesores que forman parte de la plantilla de las licenciaturas en Ciencias de la Comunicación. Para el caso de los docentes se consideraron todas las opiniones, siendo hasta la aplicación de las entrevistas cuando se toman en cuenta las características de grupos extremos.

De los resultados obtenidos en el cuestionario se obtuvieron temas de común y temas de confrontación entre las posturas de los alumnos y los docentes; entre los primeros destacan la percepción de la educación a distancia, misma que se considera como aprendizaje autónomo y de auto estudio; mientras que en el uso de la plataforma, se percibe sólo como un repositorio de materiales y no como un espacio en el que confluyen docentes y alumnos para desarrollar el aprendizaje.

Por lo que respecta a los temas de confrontación, destacan las categorías: Retroalimentación (los docentes consideran que la retroalimentación que otorgan es suficiente; los alumnos consideran que es insuficiente); Comunicación (los docentes consideran que al alumno no le interesa comunicarse y que sólo desean obtener una calificación aprobatoria; los alumnos señalan que el docente no comunica y sólo coloca una calificación final) y Recursos didácticos (los alumnos consideran que los textos de lectura son excesivos; los docentes señalan que la lectura es fundamental para desarrollar el aprendizaje). En la Tabla 1 se detallan las categorías de análisis y la valoración de éstas de acuerdo con las posturas de docentes y alumnos. 
DOI: $10.12957 /$ teias. $\%$ Y.53660

Tabla 1: Matriz de resultados. Valoración de rasgos en común vs rasgos en conflicto DocentesAlumnos

\begin{tabular}{|c|c|c|c|c|}
\hline Categorías & Subcategorías & Docentes & Alumnos & Valoración \\
\hline $\begin{array}{l}\text { Educación a } \\
\text { distancia }\end{array}$ & Características & $\begin{array}{l}\text { Aprendizaje } \\
\text { autónomo }\end{array}$ & $\begin{array}{l}\text { Aprendizaje } \\
\text { autónomo. } \\
\text { Flexibilidad en } \\
\text { tiempo. }\end{array}$ & $\begin{array}{l}\text { Tema en } \\
\text { común }\end{array}$ \\
\hline $\begin{array}{l}\text { Percepción de la } \\
\text { plataforma. }\end{array}$ & Uso & $\begin{array}{l}\text { Es un repositorio } \\
\text { de materiales } \\
\text { Hay limitaciones, } \\
\text { no tenemos } \\
\text { permisos para } \\
\text { administrarla. } \\
\text { Hay materiales } \\
\text { ilegibles. }\end{array}$ & $\begin{array}{c}\text { Es considerada como } \\
\text { un repositorio de } \\
\text { materiales. } \\
\text { Hay ligas rotas }\end{array}$ & $\begin{array}{l}\text { Tema en } \\
\text { común }\end{array}$ \\
\hline $\begin{array}{l}\text { Estrategias } \\
\text { didácticas }\end{array}$ & Recursos & $\begin{array}{c}\begin{array}{c}\text { Lecturas, libros } \\
\text { películas }\end{array} \\
\begin{array}{c}\text { Bibliotecas } \\
\text { digitales, revistas y } \\
\text { diarios digitales. }\end{array}\end{array}$ & $\begin{array}{l}\text { Videos gráficos, } \\
\text { audios, multimedia }\end{array}$ & $\begin{array}{c}\text { Tema de } \\
\text { confrontación }\end{array}$ \\
\hline Comunicación & Redes sociales & $\begin{array}{l}\text { Al alumno no le } \\
\text { interesa } \\
\text { comunicarse, sólo } \\
\text { quiere una } \\
\text { calificación. } \\
\text { Son distractores } \\
\text { que dificultan el } \\
\text { aprendizaje }\end{array}$ & $\begin{array}{l}\text { El profesor no } \\
\text { responde a las } \\
\text { preguntas, al finalizar } \\
\text { el semestre sólo } \\
\text { califica. } \\
\text { Facebook y } \\
\text { WhatsApp } \\
\text { permitirían mejorar la } \\
\text { comunicación. }\end{array}$ & $\begin{array}{c}\text { Tema de } \\
\text { confrontación }\end{array}$ \\
\hline \multirow[t]{2}{*}{ Retroalimentación } & Opinión & $\begin{array}{l}\text { Es suficiente } \\
\text { Insuficiente }\end{array}$ & Es insuficiente & \multirow[t]{2}{*}{$\begin{array}{c}\text { Tema de } \\
\text { confrontación. }\end{array}$} \\
\hline & $\begin{array}{l}\text { Propuesta de } \\
\text { mejora }\end{array}$ & $\begin{array}{l}\text { La lectura es } \\
\text { prioritaria para }\end{array}$ & $\begin{array}{l}\text { Disminución de } \\
\text { lecturas. Mayor }\end{array}$ & \\
\hline
\end{tabular}


DOI: $10.12957 /$ teias. $\%$ Y.53660

\begin{tabular}{|c|c|c|c|c|}
\hline Categorías & Subcategorías & Docentes & Alumnos & Valoración \\
\hline $\begin{array}{c}\text { Empleo de } \\
\text { Recursos } \\
\text { didácticos }\end{array}$ & & $\begin{array}{c}\text { alcanzar el } \\
\text { aprendizaje }\end{array}$ & $\begin{array}{c}\text { empleo de recursos } \\
\text { multimedia. }\end{array}$ & $\begin{array}{c}\text { Tema de } \\
\text { confrontación }\end{array}$ \\
& & $\begin{array}{c}\text { Aumento de } \\
\text { actividades } \\
\text { presenciales. }\end{array}$ & $\begin{array}{c}\text { Mejor preparación de } \\
\text { docentes en el uso de } \\
\text { tecnologías. }\end{array}$ & \\
& & $\begin{array}{c}\text { Mayor motivación, } \\
\text { retroalimentación de } \\
\text { profesores. }\end{array}$ & \\
& & & & \\
& & & \\
& & & \\
\end{tabular}

Fuente: Elaboración propia

\section{FASE 2: ENTREVISTAS A DOCENTES}

Como parte de una segunda etapa, se llevó a cabo la aplicación de entrevistas a docentes en forma presencial, para identificar la percepción que tienen de los materiales didácticos que se encuentran en la plataforma, así como para exponer las estrategias didácticas que desarrollan fuera de la plataforma.

Las respuestas que arrojó el cuestionario sirvieron de base para la elaboración del guión de la entrevista aplicada. Para ello, se retoman los temas: aprendizaje autónomo, motivación, comunicación y, retroalimentación. Entre las preguntas que se aplicaron a los docentes se encuentran: ¿Qué estrategias didácticas utiliza para motivar a sus alumnos a aprender? ¿son suficientes los materiales didácticos que provee la plataforma para que los estudiantes desarrollen situaciones de aprendizaje? ¿A qué dificultades se ha enfrentado como docente en el SUAyED? ¿es suficiente la retroalimentación que da a sus alumnos para que ellos se apropien de los aprendizajes? ¿Qué estrategias emplea para establecer comunicación con sus alumnos?

\section{Desarrollo de las entrevistas}

Las entrevistas fueron aplicadas a siete docentes con características diversas, para ello, se retomó el ciclo de vida docentes de Huberman (1990) y se construyó el caso de la siguiente manera: docentes nóveles (jóvenes con poca experiencia en la docencia), docentes consolidados (con más de diez años en la docencia) y docentes experimentados o de salida (con más de 20 años en la docencia). Para integrar la narrativa de los testimonios y con el fin de fin de guardar el anonimato de los docentes, se construyeron etiquetas de la siguiente manera: Docente (D), Hombre (H), Mujer (M), Novel (N), Consolidado (C) y Experimentado (E). A manera de síntesis, sólo se presentan aquí algunos de los testimonios de los docentes considerados como los más relevantes:

"Los contenidos en el Sistema a Distancia son bastante ligeros, siento que se procura dar todo digerido, no hay fundamentos teóricos suficiente como para mandarlos [a los alumnos] a resolver un problema o realizar alguna actividad porque todavía no tienen elementos teóricos". (DMC)

"Creo que tenemos que ser críticos en ese sentido e identificar si los contenidos responden a los objetivos, si el objetivo está bien estructurado es probable vaya de la mano con lo que se busca". (DHC)

"Los materiales de las plataformas presentan problemas como ligas rotas, páginas desactualizadas y materiales ilegibles y en su mayoría, se trata sólo de textos, que impiden 
que los objetivos del programa se cumplan, por lo que como docentes tenemos que emplear recursos adicionales para subsanar esas deficiencias del sistema". (DHC)

"A mí me gusta mucho aplicar esta estrategia con mis alumnos. Lo primero es contactarlos, a través del chat, lo hago por grupos de diez para que sea más manejable. Una vez reunidos, asignamos los roles, a los alumnos se les dan diferentes responsabilidades vinculadas con lo que consideramos sus fortalezas. Así, por ejemplo, un alumno que a lo largo del curso demostró tener una buena redacción deberá calificar ese rubro, el que tenga buena ortografía califica ese aspecto, y así sucesivamente con cada uno de los aspectos". (DMN)

"Los materiales representa todo un reto porque además de que hay una gran cantidad de lecturas en la plataforma, y en ocasiones no se especifican capítulos o páginas, es muy común que [las lecturas] no se encuentren en los sitios indicados y eso es un problema para los estudiantes que no paran de preguntar ¿dónde puedo encontrar las lecturas?” (DME)

"Sigo encontrando algunas cuestiones que, dada mi experiencia, veo como el desarrollo de las materias que hacen, hay materias que tienen vacíos y que realizan especialistas que no tienen mucha relación, son muchas lecturas y yo veo que los alumnos se pierden mucho, tenemos que buscar alternativas para mantenerlos activos" (DHN).

En los testimonios narrados por los docentes se identificaron diferentes posturas; mientras que los docentes nóveles consideran como preponderante el uso de recursos tecnológicos en la modalidad a distancia, también reconocen la necesidad de reforzar la comunicación con los alumnos. En tanto que en el caso de los docentes consolidados se identificó la necesidad del reconocimiento académico por parte de las autoridades administrativas; asimismo, se identificó como apremiante trabajar en una resignificación de la didáctica. A su vez, se reconoció la necesidad de que el alumno SUAyED se convierta en el centro del proceso educativo adquiriendo un rol activo, crítico, cuestionador y, sobre todo, responsabilizándose de su propio aprendizaje.

En los testimonios descritos fue posible apreciar cómo, sin importar el ciclo de vida docente en el que se encuentran (noveles, consolidados y experimentados), ni su género (hombre y mujer), los docentes coincidieron en que existen vacíos en los contenidos de las asignaturas que imparten; asimismo, advierten excesos en lecturas y una sobrecarga conceptual.

Por otra parte, los testimonios de los docentes permitieron identificar las estrategias didácticas que emplean en su ejercicio profesional, entre estas se encuentran:

\section{Foros y preguntas detonadoras.}

"[...] Los foros son espacios de comunicación formados por cuadros de diálogos que pueden ir clasificados temáticamente" (PÉREZ, 2009), a su vez las preguntas detonadoras son cuestionamientos que el docente realiza con el fin de propiciar la reflexión de los estudiantes. Esta estrategia permite que los alumnos puedan realizar nuevas aportaciones, aclarar otras y refutar las de los demás participantes.

\section{Redes Sociales}

“[...] Espacio en el que los individuos interactúan con otros participantes mediante el uso de internet, se basa en software que permite el intercambio de información” (GÓMEZ, 2016); las redes sociales permiten la integración y generan un sentido de pertenencia.

\section{Trabajo colaborativo y Juego de roles}

“[...] Es una estrategia didáctica que forma parte del trabajo colaborativo que consiste en asignar al alumno una investigación y un análisis de un determinado tema" (DELGADO, 2009), esta estrategia permite que el alumno reconozca sus fortalezas escolares. 


\section{Metodología Gunawardena}

“[...] Estrategia que impulsa el trabajo colaborativo en ambientes virtuales, se basa en la corroboración y busca que los participantes lleguen a acuerdos por sí solos" (GUNAWARDENA, LOWE, 1997). Se trata de un recurso lúdico que impulsa la partición del alumno.

\section{Video Educativo}

"[...] Medio técnico audiovisual de mayor proyección que permite desarrollar la actitud crítica y mejorar el acceso a los significados: palabra, imagen y sonido" (GARCÍA, 2014). Esta estrategia permite mantener el contacto visual y auditivo y generar la confianza necesaria en el alumno para facilitar la apropiación de los aprendizajes.

A continuación en la figura 1 se presenta una matriz que concentra las estrategias didácticas que de acuerdo con los testimonios presentados, emplean los docentes que participaron en las entrevistas.

\section{Figura 1: Matriz concentradora de estrategias didácticas identificadas}

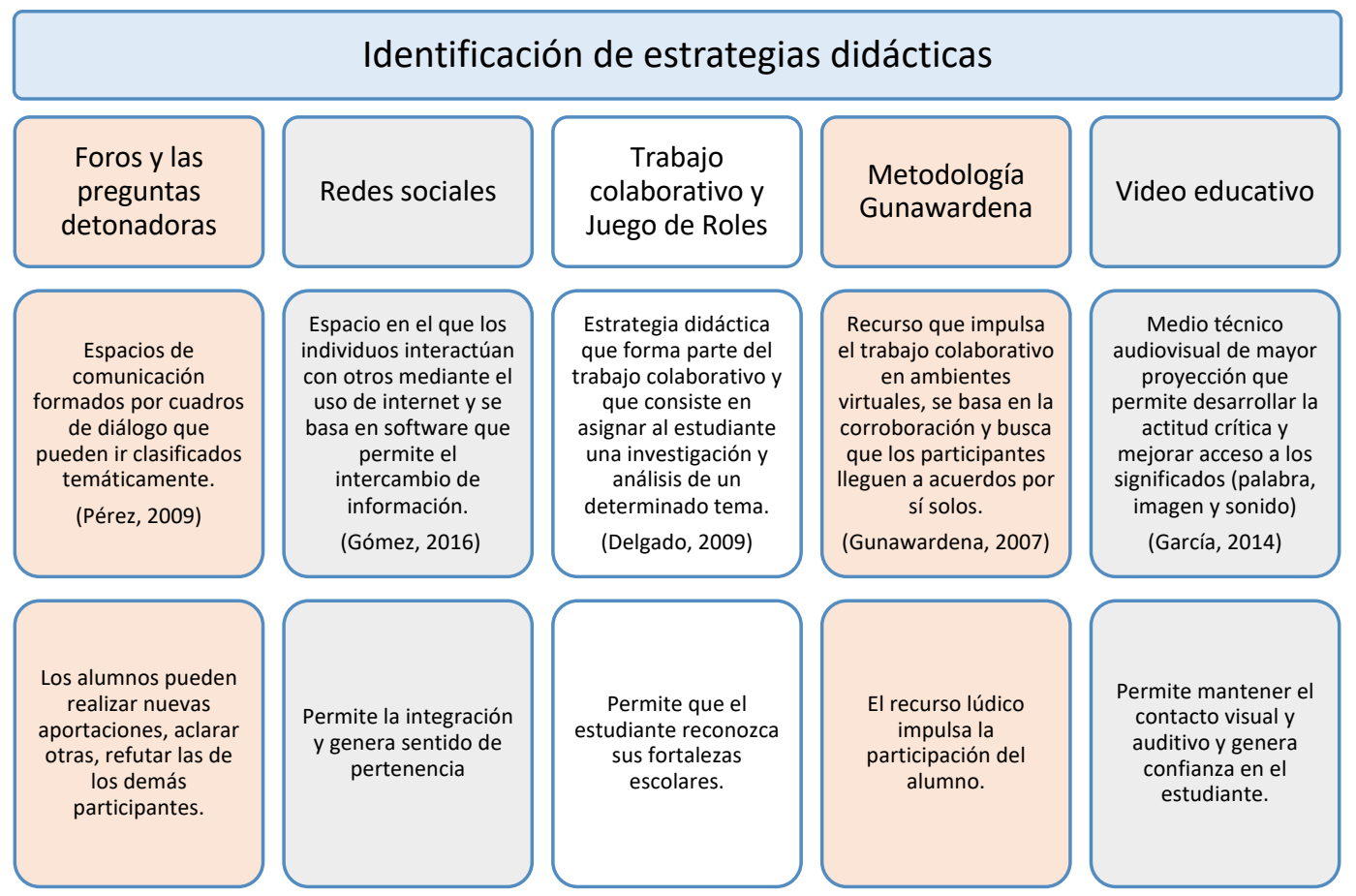

Fuente: Elaboración propia

\section{CONCLUSIONES}

Como se ha podido apreciar, la transición del trabajo docente del sistema presencial a la educación a distancia implica múltiples cambios en la forma de trabajo. Aquí, se han expuesto los principales puntos de confrontación que docentes y alumnos del SUAyED han tenido que enfrentar en un sistema educativo a distancia.

Se advierte que estas dificultades no son propias de una falta de preparación académica, sino que provienen de la falta de una construcción curricular adecuada para la educación a distancia, considerando las exigencias y necesidades propias de los entornos virtuales de aprendizaje. 
Los testimonios identificaron estrategias didácticas que los docentes emplean fuera de la plataforma para conseguir que los alumnos se apropien de los aprendizajes; no obstante, ni en las respuestas de los alumnos en los cuestionarios, como tampoco en las entrevistas, se advierte que los aprendizajes puedan ser aplicados en el contexto real en el que viven los alumnos.

Los resultados muestran que, pese a que en el SUAyED de la UNAM se trabaja, desde hace más de tres lustros a través de la educación mediada por tecnología, y a lo largo de estos años, se ha intentado reconfigurar el rol del docente, no se ha logrado construir una didáctica exprofeso para este ambiente de aprendizaje, y sólo se han trasladado las formas convencionales de la educación presencial a la educación a distancia, por ello, se advierte la necesidad de avanzar hacia un currículo centrado en temas y problemas que permita construir un Plan de Estudios organizado a partir de problemas subordinados a las disciplinas. Para ello, se requiere trabajar aún en los cambios que demanda una planeación didáctica que responda a las necesidades que implica transitar de una clase frontal a un modelo educativo de multimedios.

\section{AGRADECIMIENTOS}

La autora agradece al Posgrado en Educación de la UATx y al CONACYT por el apoyo económico para el desarrollo de este trabajo bajo la beca 861583.

\section{REFERENCIAS}

ANDRADE, Norma et al. Modelo Educativo del Sistema Universidad Abierta y Educación a Distancia de la UNAM. Distrito Federal: CUAED-UNAM, 2014. Disponible en http://www.cuaed.unam.mx/consejo/interiores/MODELO_SUAYED.pdf Consultado 10 jun. 2020.

CABERO, Julio. Reflexiones educativas sobre las tecnologías de la información y la comunicación (TIC). Tecnología, Ciencia y Educación. España: Universidad de Sevilla, n. 1, p. 19-27, abr. 2015.

COBO, Cristobal; MORAVEC, Jhon. Aprendizaje Invisible. Hacia una nueva ecología de la educación. Colección Transmedia XXI. Barcelona: Laboratrori de Mitjans Interactius/Publicacions, 2011. Disponible en http://editorialrazonypalabra.org/pdf/palabraotros/AprendizajeInvisible.pdf. Consultado 15 may. 2020.

DÍAZ BARRIGA, Ángel. De la integración curricular a las políticas de innovación en la educación superior mexicana. Perfiles Educativos. México: UNAM-ISSUE/Universia, v. XLII, n. 169, p. 160179, jul./sep. 2020. Disponible en https://doi.org/10.22201/iisue.24486167e.2020.169.59478. Consultado 30 jul. 2020.

DÍAZ BARRIGA, Ángel. Tecnologías en el aula, una experiencia en la UNAM de trabajo con tabletas electrónicas en el bachillerato. En: Consejo Mexicano de Investigación Educativa A.C., 2017, San Luis Potosí, Memoria de Simposio. San Luis Potosí: COMIE, 2017. p. 14-23. Disponible en http://www.comie.org.mx/congreso/memoriaelectronica/v14/doc/simposios/1049.pdf. Consultado 2 jun. 2020.

DÍAZ BARRIGA, Ángel. TIC en el trabajo del aula. Impacto en la planeación didáctica. Revista Iberoamericana de Educación Superior (RIES). México: UNAM-ISSUE/Universia, v. IV, n. 10, p. 3-21, jun./sep. 2013. Disponible en http://www.redalyc.org/articulo.oa?id=299128588003. Consultado 28 may. 2020. 
DOI: $10.12957 /$ teias. $\%$ Y.53660

DOWNING, Steven; HALADYNA, Thomas. Handbook of test development. New Jersey: Lawrence
Erlbaum
Associates,
2006.
Disponible

en

https:// fatihegitim.files.wordpress.com/2014/03/hndb-t-devt.pdf. Consultado 3 may. 2020.

FREINET, Celestin. Ou en sont nos techniques? Ou en est notre pédagogie. L'éducateur. Cannes: Institut Coopératif De L'école Moderne, a. 35, n. 11, p. 1-7, mar. 1963. Disponible en https://www.icem-freinet.fr/archives/educ/62-63/11-mars63/educ 11 62-63.pdf. Consultado 8 may. 2020.

GARCÍA, Lorenzo. Claves para la educación. Actores, agentes y escenarios en la sociedad actual. Madrid: Narcea, 2009.

GARCÍA, Lorenzo. De la educación a distancia a la educación virtual. Barcelona: Ariel, 2007.

GARCÍA, Lorenzo. Hacia una definición de educación a distancia. Boletín Informativo de la Asociación Iberoamericana de Educación Superior a distancia. Barcelona: UNED, a. 4, n. 18, p. 4, abr. 1987. Disponible en https://www2.uned.es/catedraunescoead/articulos $/ 1987 /$ hacia $\% 20$ una $\% 20$ definicion $\% 20$ de $\% 20$ educacion $\% 20 \mathrm{a} \% 20$ distancia.pdf. Consultado 14 feb. 2021.

GARCÍA, Manuel. Uso Instruccional del video didáctico. Revista de Investigación. Caracas, Venezuela: Universidad Pedagógica Experimental Libertador, v. 38, n. 81, p. 43-67, ene./abr. 2014. Disponible en https://www.redalyc.org/pdf/3761/376140396002.pdf. Consultado 15 jun. 2020.

GERVER, Nome. Crear hoy la escuela del mañana: la educación y el futuro de nuestros hijos. España: Fundación Santa María-Ediciones SM, 2012.

GÓMEZ, Martha; CONTRERAS, Leticia; GUTIÉRREZ, Delia. El impacto de las tecnologías de la información y la comunicación en estudiantes de ciencias sociales: un estudio comparativo de dos universidades públicas. Innovación educativa. México: IPN, v. 16, n. 71, p. 61-80, may./ago. 2016. Disponible en http://www.scielo.org.mx/scielo.php? script=sci arttext\&pid=S166526732016000200061\&lng=es\&nrm=iso. Consultado 5 jun. 2020.

GUNAWARDENA, Charlotte; LOWE, Constance; ANDERSON, Terry. Analysis of a global online debate and the development of an interaction analysis model for examining social construction of knowledge in computer conferencing. Journal of Educational Computing Research. New York: Baywood Publishing Compañy, Inc., v. 17, n. 4, p. 397-432, ene. 1997. Disponible en https://journals.sagepub.com/doi/10.2190/7MQV-X9UJ-C7Q3-NRAG. Consultado 11 abr. 2020.

HUBERMAN, Michael. Las fases de la profesión docente. Ensayo de descripción y previsión. Qurriculum: Revista de teoría, investigación y práctica educativa. España: Universidad de la Laguna, n. 2, p. 139-159, nov. 1999. Disponible en https://dialnet.unirioja.es/servlet/articulo?codigo $=3607962$. Consultado 25 abr. 2020.

KLEINING, Gerhard. Qualitativ-heuristische Sozialforschung: Schriften zur Theorie und Praxis. Hamburg: Fechner, 1994.

LANSKSHEAR, Charlotte; KNOBEL, Michelle. Problemas asociados a la metodología de la investigación cualitativa. Perfiles educativos. México: UNAM, v. XX, n. 87, p. 6-27, ene./mar. 2000. Disponible en https://www.iisue.unam.mx/perfiles/articulo/2000-87-problemas-asociados-conla-metodologia-de-la-investigacion-cualitativa.pdf. Consultado 13 may. 2020.

PÉREZ, Lourdes. El foro virtual como espacio educativo: propuestas didácticas para su uso. España: Facultad de Educación-Universidad de Salamanca, 2009. Disponible en 
DOI: $10.12957 /$ teias. $\%$ Y.53660

http://www.quadernsdigitals.net/datos web/hemeroteca/r 1/nr 662/a 8878/8878.html. Consultado 10 may. 2020.

PERRENOUD, Philippe. Diez, nuevas competencias para enseñar. Invitación al viaje. México: Grao, Colofón, 2007.

PRENSKY, Marc. Digital natives, Digital Inmigrants. On the Orizon. Lincoln: NCB University Press, v. 9, n. 5, p. 1-6, oct. 2001.

SÁNCHEZ, Abigail. Necesidades de formación de los docentes de las licenciaturas del SUAyED UNAM para la operación de sus programas. En: Congreso Internacional de Educación: Currículum, 2019, Tlaxcala, Memoria: Debates en Evaluación y Curriculum. Tlaxcala: IISUE, 2019. a. 5, n. 5. Disponible en https://posgradoeducacionuatx.org/pdf2019/E215.pdf. Consultado 1 jun. 2020.

TAYLOR, Steve; BOGDAN, Robert. Introducción a los métodos cualitativos de investigación. España: Ediciones Paidós, 1984.

ZAPATA, Miguel. Los MOOC en la crisis de la Educación Universitaria. Docencia, diseño y aprendizaje. España: CreateSpace Independent Publishing Platform, 2014.

\section{Informações da autora}

Adriana Leticia Díaz Manrique

Universidad Autonóma de Tlaxcala

E-mail: adrianadiazm@politicas.unam.mx

ORCID: https://orcid.org/0000-0001-9449-5697

Link Lattes: https://sites.google.com/politicas.unam.mx/didactica-tic/página-principal 